\title{
Delay versus Energy Consumption of the IEEE 802.16e Sleep-Mode Mechanism
}

\author{
S. De Vuyst, K. De Turck, D. Fiems, S. Wittevrongel, and H. Bruneel
}

\begin{abstract}
We propose a discrete-time queueing model for the evaluation of the IEEE 802.16e sleep-mode mechanism of Power Saving Class (PSC) $I$ in wireless access networks. Contrary to previous studies, we model the downlink traffic by means of a Discrete Batch Markov Arrival Process (D-BMAP) with $N$ phases, which allows to take traffic correlation into account. The tradeoff between energy saving and increased packet delay is discussed. In many situations, the sleep-mode performance improves for heavily correlated traffic. Also, when compared to other strategies, the exponential sleep-period update strategy of PSC I may not always be the best.
\end{abstract}

Index Terms-Discrete-time queueing model, sleep mode, IEEE 802.16e, analytic study.

\section{INTRODUCTION}

W E focus on the performance of the sleep-mode mechanism for Power Saving Class (PSC) I, defined in the IEEE 802.16e standard for Broadband Wireless Access networks [1]. This standard aims to support mobile users in WiMAX (IEEE 802.16) wireless networks by addressing typical mobility-related issues. On the MAC layer, these include enhanced security, faster handover protocols and energy saving mechanisms, the latter of which are the topic of this letter.

Consider the downstream communication between a single Mobile Station (MS) and its serving Base Station (BS) over a WiMAX wireless link. To extend its battery life, the IEEE 802.16e sleep-mode mechanism enables the MS to turn off its radio interface for a certain time whenever the BS has no packets in its queue destined for this MS, see Fig. 1. Specifically, if the BS's queue is empty, the MS starts a sleep period during which it remains powered down and thus cannot be reached by the BS. At the end of this period, the MS is briefly reactivated (listening interval, indicated on the figure by ' $L$ ') to check whether there are packets waiting for it at the BS. If not, the MS initiates a second sleep period, a third, and so on. However, if any packets have arrived at the BS during the last sleep period, the MS remains powered and enters awake mode. The BS then transmits all the packets in its buffer exhaustively, after which the whole procedure is repeated. Hence, the BS transmitter alternates between idle periods, consisting of a number of contiguous sleep periods, and busy periods. In case of IEEE 802.16e PSC $\mathrm{I}$, an exponential update strategy is used. The first sleep period of an idle period has length $T_{0}$ while every subsequent sleep

Manuscript received October 1, 2008; revised May 14, 2009; accepted August 7, 2009. The associate editor coordinating the review of this letter and approving it for publication was Y.-B. Lin.

The authors are with the SMACS Research Group, Department of Telecommunications and Information Processing, Ghent University, St.Pietersnieuwstraat 41 Ghent B-9000, Belgium (e-mail: \{sdv, kdeturck, df, sw, hb\}@telin.ugent.be).

Digital Object Identifier 10.1109/TWC.2009.080287

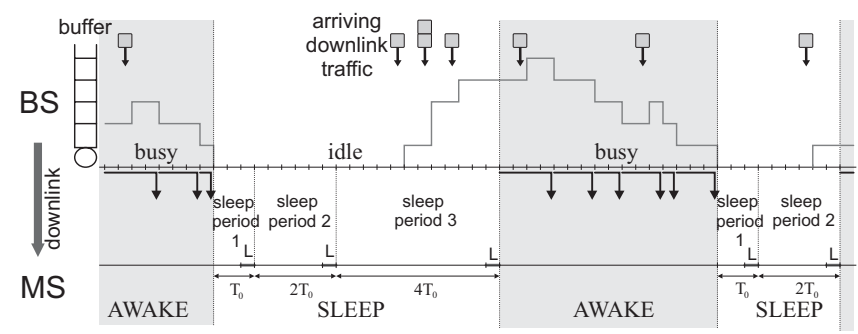

Fig. 1. Operation of the IEEE 802.16e PSC I sleep-mode mechanism in case of downlink traffic. Without loss of generality, we assume a 'sleep period'to include the ensuing listening interval ' $L$ ', contrary to the definition in the standard. This assumption facilitates the analysis, without affecting the validity of the results.

period doubles in length until a maximum $T_{\max }$ is reached. After that, all additional sleep periods have length $T_{\max }$. We denote this update strategy by $\left(T_{0}, 2 T_{0}, 4 T_{0}, \ldots, T_{\max }\right)$.

The drawback of this mechanism is an inherent degradation of the QoS. As the transmission of packets arriving at the BS must await the end of the current sleep period, the overall delay of the packets increases. It is therefore important to carefully tune the parameters of the sleep-mode mechanism in order to obtain a good tradeoff between energy reduction and additional delay. To this end, the MS energy consumption has been studied in e.g. [3], [4] for sleep mode PSC I and in [5], [6] for PSC II. Queueing models that also analyse the BS packet delay have been presented in [7]-[9]. All of these models however, assume an uncorrelated memoryless arrival process (i.e. a Poisson process) for the traffic arriving at the BS, which is very restrictive in many cases [10]. It is known that Markov-modulated models [11], [12] can capture the memory of traffic traces quite well. Hence, we consider here the sleep-mode BS queueing behaviour assuming a Discrete Batch Markov Arrival Process (D-BMAP) [13]. This is a far more general model that can capture complex correlation structures. The model proposed is also generic with respect to the update strategy of the sleep periods and not restricted to the exponential strategy of IEEE 802.16e PSC I alone. This allows us to explore some alternatives: in section $\mathrm{V}$, we compare the performance of the exponential strategy with both constant and linear strategies.

The main purpose of this letter is to demonstrate that traffic correlation heavily affects the performance of the sleep-mode mechanism, both in terms of achieved energy efficiency and packet delay. Neglecting this correlation in the analysis, as in [3]-[9], may therefore lead to a very poor estimation of the true performance characteristics. Additionally, we propose a convenient efficiency metric that can be used to optimise 
the design parameters. In section II we present our model of the BS queue. The analysis is outlined in Sections III and IV. For an arrival process with two phases, we compare the performance of different update strategies in section V. Section VI concludes.

\section{MOdeL}

We model the BS as a discrete-time single-server queue with infinite capacity and a first-come-first-served (FIFO) discipline [14]. Time is assumed to be divided into fixedlength intervals called slots. The service time of a packet is the number of slots required to transmit it successfully from the BS to the MS. We assume the service times of the packets to be independent random variables with common probability generating function (pgf) $S(z)$ and mean $\mathrm{E}[s]=S^{\prime}(1)$. This is a reasonable assumption in case the wireless medium is fast fading [15].

Packets arrive in the queue according to a D-BMAP with $N$ phases (or states) [13]. In each slot, the arrival process resides in one of the $N$ phases and is characterised by the probabilities $a(k, j \mid i)(k \geqslant 0, i, j \in\{1, \cdots, N\})$ that, given phase $i$ in a slot, there are $k$ packet arrivals and the phase switches to $j$ in the next slot. These probabilities can be arranged in the $N \times N$ probability generating matrix $(\mathrm{pgm}) \mathbf{A}(z)$ with entries

$$
[\mathbf{A}(z)]_{i j}=\sum_{k=0}^{\infty} a(k, j \mid i) z^{k} .
$$

Many methods exist to fit this model to measured traffic data, see e.g. [16]. The equilibrium distribution of the phases is found as the $1 \times N$ probability vector $\varsigma$ satisfying $\boldsymbol{\varsigma} \mathbf{A}(1)=\boldsymbol{\varsigma}$ and $\boldsymbol{\varsigma} \mathbf{1}=1$, where $\mathbf{1}$ is a $N \times 1$ column vector with all entries equal to 1 . The mean number of arrivals per slot is then $\mathrm{E}[a]=$ $\boldsymbol{\varsigma} \mathbf{A}^{\prime}(1) \mathbf{1}$ and the load of the BS queue is $\rho=\mathrm{E}[a] \mathrm{E}[s]$.

The sleep-period update strategy is $\left(t_{1}, t_{2}, \ldots\right)$ where the positive integers $t_{n}(n \geqslant 1)$ are the lengths of the subsequent sleep periods of the same idle period. Further define $\tau_{n}=$ $\sum_{i=1}^{n} t_{i}(n \geqslant 1)$ and $\tau_{0}=0$ as the starting slots of the sleep periods relative to the start of the idle period. We will assume that the sleep-period lengths remain fixed after the $J$ th sleep period, i.e. $t_{n}=t_{J}$ for $n \geqslant J$. For the PSC I mechanism, this clearly applies with $t_{J}=T_{\max }$.

\section{QUEUEING ANALYSIS}

In [17], we obtained the distribution of the BS queue content via some intermediate steps. First the queue is considered at service completion instants only, i.e. at the beginning of a slot following the transmission of a packet. If the $i$ th entry of the probability generating vector $(\mathrm{pgv}) \mathbf{U}_{c}(z)$ is the partial pgf of the queue content at a service completion with phase $i$, then

$$
\mathbf{U}_{c}(z)=\mathbf{U}_{c}(0)(\mathbf{V}(z)-\mathbf{I})(z \mathbf{I}-S(\mathbf{A}(z)))^{-1} S(\mathbf{A}(z)),
$$

where I denotes the $N \times N$ identity matrix and where $S(\mathbf{A}(z))$ is the matrix power series in $\mathbf{A}(z)$ with the same coefficients as the power series $S(z)$ in $z$. The $\operatorname{pgm} \mathbf{V}(z)$ is calculated as

$$
\begin{aligned}
\mathbf{V}(z)= & \sum_{n=1}^{J-1} \mathbf{A}(0)^{\tau_{n-1}}\left[\mathbf{A}(z)^{t_{n}}-\mathbf{A}(0)^{t_{n}}\right] \\
& +\mathbf{A}(0)^{\tau_{J-1}}\left(\mathbf{I}-\mathbf{A}(0)^{t_{J}}\right)^{-1}\left[\mathbf{A}(z)^{t_{J}}-\mathbf{A}(0)^{t_{J}}\right] .
\end{aligned}
$$

In (1), the unknown vector $\mathbf{U}_{c}(0)$ can be determined by invoking the boundedness of $\mathbf{U}_{c}(z)$ for all $|z| \leqslant 1$ using the following procedure. First, note that the queue content at subsequent service completions forms a stochastic process of the M/G/1-type, see e.g. [18]. The transform method for solving such systems has been treated extensively in [19]. Specifically, it is shown that the matrix $z \mathbf{I}-S(\mathbf{A}(z))$ on the right-hand side of (1) becomes singular in exactly $N$ points $z=z_{i}(i=1, \ldots, N)$ in the unit disc, counting multiplicities. These points are the zeroes of the function $\operatorname{det}(z \mathbf{I}-S(\mathbf{A}(z)))$ and can be calculated numerically, along with their multiplicities, using a root-finding algorithm. In case the zeroes $z_{i}$ are distinct, i.e. all with multiplicity one, it is possible to construct column vectors $\boldsymbol{\xi}_{i}$ satisfying

$$
\left(z_{i} \mathbf{I}-S\left(\mathbf{A}\left(z_{i}\right)\right)\right) \boldsymbol{\xi}_{i}=\mathbf{0},
$$

for each $i=1, \ldots, N$. Clearly, as the matrix $z_{i} \mathbf{I}-S\left(\mathbf{A}\left(z_{i}\right)\right)$ is singular, a non-trivial solution $\boldsymbol{\xi}_{i}$ always exists. Now, note that in (1) the last two factors commute. Substitution of $z=z_{i}$ in (1) and post-multiplying both sides with (3) yields

$$
\mathbf{U}_{c}(0)\left(\mathbf{V}\left(z_{i}\right)-\mathbf{I}\right) \boldsymbol{\xi}_{i}=0, \quad i=1, \ldots, N .
$$

This is a set of $N$ equations that are known to be independent [20] and thus allow to solve for the entries of $\mathbf{U}_{c}(0)$. The procedure is somewhat more involved in the peculiar case that zeroes $z_{i}$ occur with multiplicity larger than one, see [19].

Once the distribution of the queue content at service completions (1) is known, it is possible to obtain the distribution for arbitrary slot as well. Let the $i$ th entry of the $\operatorname{pgv} \mathbf{U}(z)$ be the partial pgf of the queue content in an arbitrary slot with phase $i$. In [17], we find

$$
\mathbf{U}(z)=(z-1) \mathrm{E}[a] \mathbf{U}_{c}(z)(\mathbf{A}(z)-\mathbf{I})^{-1} .
$$

Additionally, if $u_{c, 0}=\mathbf{U}_{c}(0) \mathbf{1}$ is the probability that the queue is empty at a service completion, the mean length of a busy period is $\mathrm{E}[B]=\mathrm{E}[s] / u_{c, 0}$, whereas the mean length of an idle period is found as

$$
\begin{aligned}
\mathrm{E}[I]=\frac{\mathbf{U}_{c}(0)}{u_{c, 0}}\left[\sum_{n=1}^{J-1} t_{n} \mathbf{A}(0)^{\tau_{n-1}}\right. & \\
& \left.+t_{J} \mathbf{A}(0)^{\tau_{J-1}}\left(\mathbf{I}-\mathbf{A}(0)^{t_{J}}\right)^{-1}\right] \mathbf{1},
\end{aligned}
$$

\section{MeAn DELAY AND ENERGy CONSUMPTION}

Many interesting characteristics of the distribution of the BS queue content can be derived from (4), such as the moments up to any order or its tail asymptotics. For instance, the mean queue content is $\mathrm{E}[u]=\mathbf{U}^{\prime}(1) \mathbf{1}$ and, by Little's law [21], the mean delay experienced by an arbitrary packet is $\mathrm{E}[d]=$ $\mathrm{E}[u] / \mathrm{E}[a]$. With regard to the energy consumption of the MS, we assume that the MS is active during every busy slot, as well as during the last slot of each sleep period (i.e. the listening interval). When active, it consumes one unit of energy, while no energy is used during the other (idle) slots. Hence, the mean packet delay $D$ and the energy $E$ consumed by the MS per slot are

$$
D=\frac{\mathbf{U}^{\prime}(1) \mathbf{1}}{\mathrm{E}[a]} \quad \text { and } \quad E=\rho+(1-\rho) \frac{\gamma}{\mathrm{E}[I]},
$$


where $\gamma$ denotes the mean number of subsequent sleep periods in a single idle period,

$$
\gamma=\frac{\mathbf{U}_{c}(0)}{u_{c, 0}}\left[\sum_{n=1}^{J-1} \mathbf{A}(0)^{\tau_{n-1}}+\mathbf{A}(0)^{\tau_{J-1}}\left(\mathbf{I}-\mathbf{A}(0)^{t_{J}}\right)^{-1}\right] \mathbf{1} .
$$

\section{EXAMPLES AND DISCUSSION}

These results can be used to assess the performance of the sleep-mode mechanism under a wide variety of traffic conditions, service-time distributions and sleep-period update strategies. Some important phenomena can be demonstrated with as little as two phases for the D-BMAP arrival process. We here assume an ON/OFF traffic source:

$$
\mathbf{A}(z)=\left[\begin{array}{cc}
1-q+q z & 0 \\
0 & 1
\end{array}\right]\left[\begin{array}{cc}
p_{1} & 1-p_{1} \\
1-p_{2} & p_{2}
\end{array}\right] .
$$

The second matrix contains the phase transition probabilities. The first matrix is chosen such that when a slot is in phase $1(\mathrm{ON})$, an arrival occurs with probability $q$, whereas there are $n o$ arrivals in a slot with phase 2 (OFF). The equilibrium distribution of the phases directly follows as $\varsigma=\left[\begin{array}{ll}\sigma & 1-\sigma\end{array}\right]$ where $\sigma=\frac{1-p_{2}}{2-p_{1}-p_{2}}$, which means that the long-run fraction of slots in the ON- and OFF-phase is $\sigma$ and $1-\sigma$ respectively. Also, the mean numbers of contiguous slots in the same phase are $\frac{K}{1-\sigma}$ and $\frac{K}{\sigma}$ respectively, with $K=\frac{1}{2-p_{1}-p_{2}}$. Hence, higher values of $K$ mean that the arrival process remains in the same phase for longer periods of time and therefore exhibits more correlation. If $K=1$ however, the arrivals are uncorrelated. The parameter $K$ is referred to as the correlation factor or the burstiness factor of the arrival process [22]. Let us assume ARQ retransmissions are used to make sure a packet is received correctly by the MS. If each transmission requires one slot and succeeds with probability 0.5 , then the 'service times' of the BS queue have a shifted geometric distribution with mean $\mathrm{E}[s]=2$. The load of the system is then $\rho=q \sigma \mathrm{E}[s]$. Three types of strategies for updating the consecutive sleepperiod lengths are considered: constant, denoted as $\left\langle T_{0} \mathrm{C}\right\rangle$, where every sleep period has the same length $T_{0}$; linear, denoted as $\left\langle T_{0} \mathrm{~L}\right\rangle=\left(T_{0}, 2 T_{0}, 3 T_{0}, 4 T_{0}, \ldots, T_{\max }\right)$, where each sleep period increases with the same amount $T_{0}$ up to a maximum $T_{\max }$; exponential, where the subsequent sleep periods double in length up to maximum $T_{\max }$ and which we denote as $\left\langle T_{0} \mathrm{E}\right\rangle=\left(T_{0}, 2 T_{0}, 4 T_{0}, 8 T_{0}, \ldots, T_{\max }\right)$. For the linear and exponential strategies, we assume $T_{\max }=256$ slots.

Use of the sleep-mode mechanism increases both the packet delay (which is bad) and the battery life of the MS (which is good). To make a fair tradeoff, one needs to compare both delay and energy consumption to the reference case where the MS is always activated. Let $D^{*}$ and $E^{*}=1$ be the mean packet delay and MS energy consumption in this reference case (they can be obtained from (6) using strategy $\langle 1 \mathrm{C}\rangle$ ). We define

$$
\beta_{E}=\frac{E^{*}}{E}-1, \quad \text { and } \quad \beta_{D}=\frac{D}{D^{*}}-1,
$$

which respectively give the battery-life extension and the delay increase resulting from sleep-mode usage. The minimal value of both $\beta_{E}$ and $\beta_{D}$ is equal to 0 , corresponding to the reference case of communication without the sleep-mode mechanism. However, in case any of the above sleep-mode

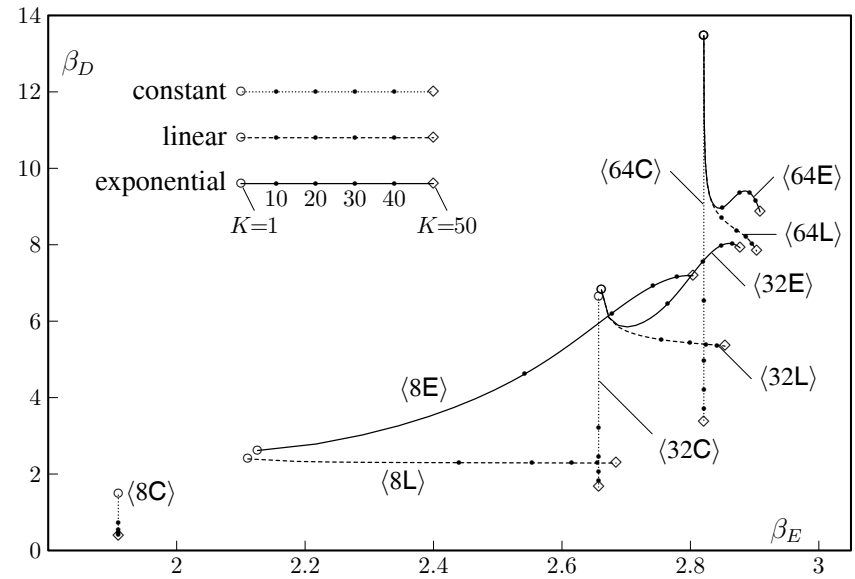

Fig. 2. Shift of $\left(\beta_{E}, \beta_{D}\right)$ vs. $K$ in case $\sigma=0.25, \rho=0.25$ and different strategies.

strategies is used, both values will increase. For example, $\beta_{E}=0.7$ would indicate that the MS's battery life is increased by $70 \%$ while the corresponding $\beta_{D}>0$ measures the inherent delay cost.

In Fig. 2 we illustrate how the traffic correlation $K$ typically affects the performance of various sleep-period update strategies. Here, $\sigma$ and $\rho$ are both fixed at 0.25 and we show the shift of the point $\left(\beta_{E}, \beta_{D}\right)$ as $K$ increases from 1 (no traffic correlation) to 50 (high correlation). Clearly, points in this graph represent a better performance as they are situated lower (small $\beta_{D}$ ) and further to the right (high $\beta_{E}$ ). First observe that for the constant strategies, the energy consumption is insensitive to $K$. In fact, for $\left\langle T_{0} \mathrm{C}\right\rangle$ we know that $E=\rho+(1-\rho) \frac{1}{T_{0}}$ because exactly one out of every $T_{0}$ idle slots is a listening slot. However, the delay cost $\beta_{D}$ is reduced significantly if $K$ increases, especially so when the sleep period $T_{0}$ is larger. For the linear and exponential strategies, the situation is less unambiguous, although increased traffic correlation generally allows more energy to be saved (especially for low initial sleep period $T_{0}$ ). Sometimes, but not necessarily, this comes at the price of a higher delay. The question which strategy performs 'optimally' for a given traffic pattern, is obviously a matter of how much energy saving is valued over delay cost. For example, let us compare the shift of $\langle 8 \mathrm{E}\rangle$ and $\langle 64 \mathrm{C}\rangle$ in Fig. 2 in case of a delay-sensitive application. For $K=1,\langle 8 \mathrm{E}\rangle$ obviously performs best, as its delay cost is about six times lower. For $K=50$ however, the delay of $\langle 64 \mathrm{C}\rangle$ is only half that of $\langle 8 \mathrm{E}\rangle$ while both strategies have almost the same energy consumption.

Whether or not correlation is beneficial for a certain strategy also depends on the load $\rho$, as we demonstrate in Fig. 3. The shift of $\left(\beta_{E}, \beta_{D}\right)$ for strategies $\langle 64 C\rangle$ and $\langle 64 E\rangle$ is plotted as the load $\rho$ changes from 0.03 to 0.47 for three values of the traffic correlation factor $K$. This confirms our previous observation that correlation is always beneficial for a constant strategy. For the exponential strategy however, correlation improves the performance only if the load is high, while the opposite is true in case of low load.

In order to compare different sleep-mode strategies quantitatively, we need a scalar metric for their performance. We 




Fig. 3. Shift of $\left(\beta_{E}, \beta_{D}\right)$ vs. $\rho$ in case $\sigma=0.25, K=1,10,50$ and for strategies $\langle 64 \mathrm{E}\rangle$ and $\langle 64 \mathrm{C}\rangle$.

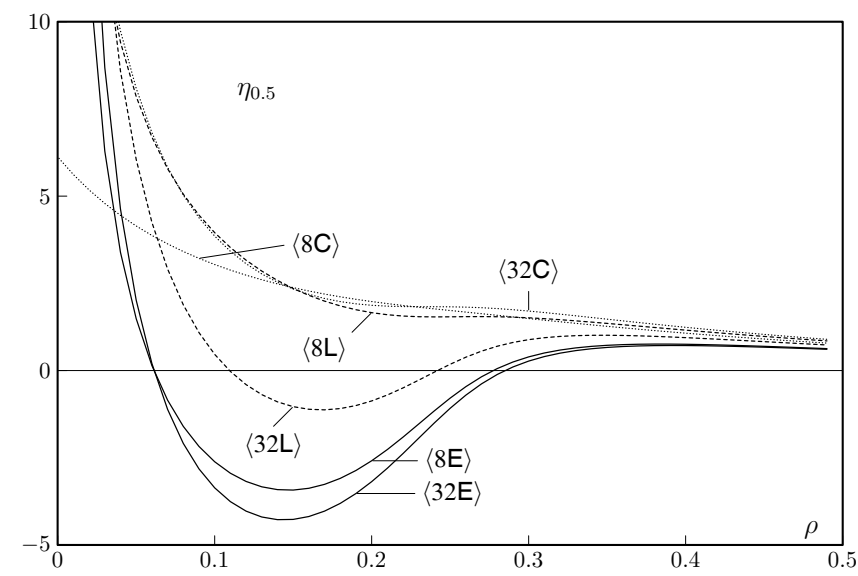

Fig. 4. Efficiency $\eta_{0.5}$ for different strategies in case $K=50, \sigma=$ 0.25 .

propose to define the efficiency $\eta_{c}$ of a strategy as

$$
\eta_{c}=\beta_{E}-c \beta_{D},
$$

where $c$ is a delay 'penalty' which reflects to what extent low packet delay is preferred over energy saving. For delaysensitive applications, this penalty must be taken relatively high. If the primary focus is to extend battery life however, then $c$ should be small. An example is given in Fig. 4 where the efficiency $\eta_{0.5}$ is shown as a function of the load in case $K=50$ and $\sigma=0.25$. According to cost function (9), it is efficient to use a sleep-mode strategy as long as its $\eta_{c}$ is positive. If $\eta_{c}<0$ however, the best solution is not to use the sleep-mode mechanism at all (or equivalently, to use $\langle 1 \mathrm{C}\rangle$ ). Particularly in Fig. 4, observe that the constant and linear strategies outperform the exponential (IEEE 802.16e) strategies for all but the smallest values of the load.

Clearly, more complex traffic models than (7) can be considered as well. For example, the 4IPP model in [23], often used in IEEE 802.16 performance studies, consists of the superposition of 4 independent ON/OFF-sources. Each of them can be described as a 2-state D-BMAP of the form (7): source $i(i=1,2,3,4)$ has $\operatorname{pgm} \mathbf{A}_{i}(z)$ as in (7) with parameters $\sigma_{i}, q_{i}$ and correlation factor (or 'timescale') $K_{i}$.
The superposition of these four sources is again a D-BMAP, with $\operatorname{pgm} \mathbf{A}(z)=\bigotimes_{i=1}^{4} \mathbf{A}_{i}(z)$, and the analysis of sections III and IV can be applied.

\section{CONCLUSIONS}

We provided an analytic framework to estimate the performance of the (IEEE 802.16e) sleep-mode mechanism under a wide range of conditions for the traffic model (D-BMAP), the service-time distribution and the sleep-period update strategy. We show that in many situations the sleep-mode mechanism performs significantly better in case of highly correlated traffic. Another important observation is the fact that the exponential sleep-period update strategy used in IEEE 802.16e PSC I is not always the best choice for certain traffic patterns between BS and MS.

\section{ACKNOWLEDGEMENTS}

We wish to thank the anonymous reviewers for their valuable comments. The third author is a postdoctoral fellow with the Research Foundation - Flanders (FWO-Vlaanderen).

\section{REFERENCES}

[1] IEEE 802.16e-2005, "Part 16: Air interface for fixed and mobile broadband wireless access systems - Amendment 2, Corrigendum 1," Feb. 2006.

[2] B. Kim, J. Park, Y.-H. Choi, "Power saving mechanisms of IEEE 802.16e: sleep mode vs. idle mode," Lecture Notes Computer Science, vol. 4331 (ISPA 2006 Ws), pp. 332-340, 2006.

[3] Y. Xiao, "Energy saving mechanism in the IEEE 802.16e wireless MAN," IEEE Commun. Lett., vol. 9, no. 7, pp. 595-597, 2005.

[4] Y. Zhang and M. Fujise, "Energy management in the IEEE 802.16e MAC," IEEE Commun. Lett., vol. 10, no. 4, pp. 311-313, 2006.

[5] Y.-H. Han, S.-G. Min, and D. Jeong, "Performance comparison of sleep mode operations in IEEE 802.16e terminals," Lecture Notes Computer Science, vol. 4490 (ICCS 2007, Part IV), pp. 441-448, 2007.

[6] J.-R. Lee, "Energy efficient sleep interval decision method considering delay constraint for VoIP traffic," Lecture Notes Computer Science, vol. 4003 (NEW2AN 2006), pp. 142-151, 2007.

[7] K. Han and S. Choi, "Performance analysis of sleep mode operation in IEEE 802.16e mobile broadband wireless access systems," in Proc. IEEE 63rd Vehicular Techn. Conf. VTC 2006-Spring, vol. 3, pp. 11411145.

[8] Y. Park and G. U. Hwang, "Performance modelling and analysis of the sleep mode in IEEE 802.16e WMAN," in Proc. IEEE 65th Vehicular Techn. Conf. VTC 2007-Spring, pp. 2801-2806.

[9] J.-B. Seo, S.-Q. Lee, N.-H. Park, H.-W. Lee, and C.-H. Cho, "Performance analysis of sleep mode operation in IEEE 802.16e," in Proc. IEEE 60th Veh. Technol. Conf. VTC 2004-Fall, vol. 2, pp. 1169-1173.

[10] V. Paxson and S. Floyd, "Wide-area traffic: the failure of Poisson modeling," IEEE/ACM Trans. Networking, vol. 3, no. 3, pp. 226-244, 1995.

[11] A. T. Andersen and B. F. Nielsen, "A Markovian approach for modeling packet traffic with long-range dependence," IEEE J. Sel. Areas Commun., vol. 16, no. 5, 1998.

[12] H. Michiel and K. Laevens, "Teletraffic engineering in a broad-band era," Proc. IEEE, vol. 85, no. 12, pp. 2007-2033, 1997.

[13] C. Blondia and O. Casals, "Statistical multiplexing of VBR sources: a matrix-analytic approach," Perform. Evaluation, vol. 16, no. 1-3, pp. 5-20, 1992.

[14] H. Bruneel, "Performance of discrete-time queueing systems," Comp. \& Oper. Res., vol. 20, no. 3, pp. 303-320, 1993.

[15] C. C. Tan and N. C. Beaulieu, "On first-order Markov modeling for the Rayleigh fading channel," IEEE Trans. Commun., vol. 48, no. 12, pp. 2032-2040, 2000.

[16] D. Fiems, V. Inghelbrecht, B. Steyaert, and H. Bruneel, "Markovian characterisation of H.264/SVC scalable video," in Proc. 15th Int. Conf. Analytical Stochastic Modelling Techniques Applications, ASMTA '08 June 2008, Nicosia, Cyprus. 
[17] K. De Turck, S. De Vuyst, D. Fiems, and S. Wittevrongel, "Performance analysis of the IEEE 802.16e sleep mode for correlated downlink traffic," Telecommun. Syst., vol. 39, no. 2, pp. 145-156, 2008.

[18] M. F. Neuts, Structured Stochastic Matrices of M/G/1 Type and Their Applications. New York: Marcel Dekker, 1989.

[19] H. R. Gail, S. L. Hantler, and B. A. Taylor, "Spectral analysis of M/G/1 and G/M/1 type Markov chains," Adv. Appl. Probab., vol. 28, no. 1, pp. 114-165, 1996.
[20] H. R. Gail and S. L. Hantler, "Linear independence of root equations for M/G/1 type Markov chains," Queueing Syst., vol. 20, no. 3-4, pp. 321-339, 1995.

[21] D. Fiems and H. Bruneel, "A note on the discretization of Little's result," Oper. Res. Lett., vol. 30, no. 1, pp. 17-18, 2002.

[22] S. Wittevrongel and H. Bruneel, "Discrete-time queues with correlated arrivals and constant service times," Comp. \& Oper. Res., vol. 26, no. 2, pp. 93-108, 1999.

[23] C. R. Baugh, J. Huang, R. Schwartz, and D. Trinkwon, "Traffic model for 802.16 TG3 MAC/PHY simulations," technical report, IEEE 802.16 Broadband Wireless Access Working Group, 2001. 